\title{
HALO FORMATION OF BUNCHED BEAMS IN PERIODIC FOCUSING SYSTEMS
}

\begin{abstract}
A. Letchford, RAL, UK, K. Bongardt, M. Abstract

A very critical design issue for high intensity proton linacs is to keep particle loss below $1 \mathrm{~W} / \mathrm{m}$ at the high energy end and allow unconstrained hand on maintenance. Particle loss is caused by a small number of particles outside the dense beam core, called the beam halo. Halo formation of bunched beams in a periodic focusing channel is driven by mismatch, high space charge and temperature anisotropy. Unstable particles oscillate in all 3 phase space planes. Mode overlap due to large mismatch amplitudes can lead to a halo much larger than seen in uniform focusing systems. Resonance crossing due to acceleration is a possibility for designing high intensity linacs.
\end{abstract}

\section{SINGLE PARTICLE - ENVELOPE RESONANCE}

For realistic particle distributions, with nonlinear space charge forces, particles inside the beam core have different tunes. Parametric particle - envelope resonances can occur between the single particle tune and the frequency of the mismatch of the oscillating beam core.

For a bunched beam the frequencies of the three eigenmodes are approximately given by [1]

$$
\sigma_{e n v, Q}=2 \sigma_{t}
$$

for the pure transverse quadrupolar mode and by

$$
\sigma_{e n v, H}^{2}=A+B, \sigma_{e n v, L}^{2}=A-B
$$

with

$$
A=\sigma_{t o}^{2}+\sigma_{t}^{2}+\frac{1}{2} \sigma_{l o}^{2}+\frac{3}{2} \sigma_{l}^{2}
$$

and

$B=\sqrt{\left(\sigma_{t o}^{2}+\sigma_{t}^{2}-\frac{1}{2} \sigma_{l o}^{2}-\frac{3}{2} \sigma_{l}^{2}\right)^{2}+2\left(\sigma_{t o}^{2}-\sigma_{t}^{2}\right)\left(\sigma_{l o}^{2}-\sigma_{l}^{2}\right)}$

for the high and low mode which couple the transverse and longitudinal directions. The mismatch modes are expressed by the full and zero current transverse and longitudinal tunes $\sigma_{t}, \sigma_{t o}, \sigma_{l}$ and $\sigma_{l o}$. For the quadrupolar mode one has for the relative mismatches

$$
\frac{\Delta a_{x}}{a_{x_{0}}}=-\frac{\Delta a_{y}}{a_{y o}}, \frac{\Delta b}{b_{o}}=0 .
$$

Here only anti-phase transverse mismatches is present. In case of the high and low mode one has

$$
\frac{\Delta a_{x}}{a_{x o}}=\frac{\Delta a_{y}}{a_{y o}}=g_{H / L} \frac{\Delta b}{b_{o}} .
$$

with

$$
g_{H / L}=\frac{\sigma_{t o}^{2}-\sigma_{t}^{2}}{\sigma_{e n v, H / L}^{2}-2\left(\sigma_{t o}^{2}+\sigma_{t}^{2}\right)} .
$$

$g_{H}$ is always positive and $g_{L}$ always negative.

The analytical expressions are derived for a uniform focusing channel with linear external and space charge forces. The derivatives of the formfactors are neglected. Numerical simulations of a periodic bunched beam transport line with spherical bunches $(b / a \sim 1)$ or elongated bunches $(b / a \sim 3)$ have shown that all three eigenmodes can be excited by using the above stated amplitude ratios.

Due to nonlinear space charge forces, particles have tunes distributed between the full current and zero current tune. The condition for exciting a parametric resonance either transversely or longitudinally is given by

$$
\frac{\sigma_{t, l}^{p}}{\sigma_{e n v}}=\frac{m}{n}=\frac{1}{2}, \frac{1}{3}, \ldots
$$

with

$$
\begin{aligned}
\sigma_{t} & \leq \sigma_{t}^{p} \leq \sigma_{t o}, \\
\sigma_{l} & \leq \sigma_{l}^{p} \leq \sigma_{l o}
\end{aligned}
$$

where $\sigma_{e n v}$ is one of the three envelope tunes of the mismatched radii and $\sigma_{t, l}^{p}$ the single particle tune.

Fig. 1 shows the halo formation excited by the quadrupolar mode with $20 \%$ initial mismatch. Results are derived from Monte Carlo simulation of an almost spherical bunched beam in a periodic focusing system [1]. All calculations discussed below were done with at least 10000 particles which interact fully in $3 \mathrm{~d}$. A $6 \mathrm{~d}$ waterbag distribution is used as input. Clearly visible is the onset of halo formation and its leveling afterwards. Above $1 \%$ of the particles are outside $10 * \epsilon_{r m s}$, compared to $0.1 \%$ for the not shown matched case. The halo is limited to about $30 * \epsilon_{r m s}$ in phase space. Similar results have been reported from Monte Carlo simulations for selfconsistent equipartitioned bunched beams in a uniform focusing channel [2]. For spherical bunches the halo formation is similar in the transverse and longitudinal directions.

\section{UNSTABLE PARTICLE MOTION IN A PERIODIC FOCUSING CHANNEL}

In a periodic focusing channel additional resonances and instabilities, which don't exist in an uniform channel, can influence single particle motion. One is the envelope - lattice instability which occurs for a bunched beam if the high mode frequency is nearly $180^{\circ}$. The instability leads to an increase of the rms beam envelope. Other important resonances can occur between single particles and the periodic 


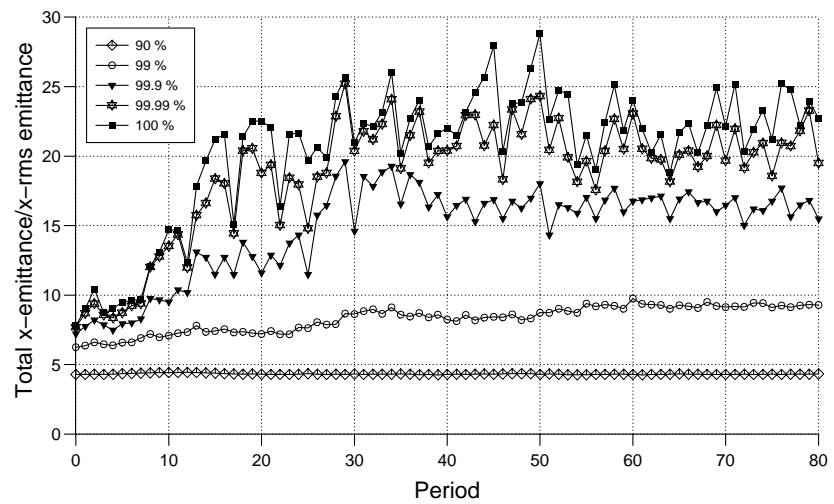

Figure 1: Transverse emittance ratio along a mismatched bunched beam transfer line

lattice if for dc [3] or bunched beams [4] the tune depressions are below 0.4 and the full current tunes below $60^{\circ}$.

Beside these instabilities other particle - lattice resonances can be excited either by temperature exchange or by mismatch. Even if all above mentioned instabilities are avoided large mismatch amplitudes can create unstable particle motion.

\subsection{Unstable Particle Motion due to Resonance Overlap}

In a periodic focusing system a parametric particle - lattice resonance is excited if the zero current tune is above $90^{\circ}$. If a particle - envelope resonance, excited by mismatch, is nearby a particle - lattice resonance, then particles can be driven by the envelope resonance into lattice resonance. This is called resonance overlap which can lead to unstable particle motion with large amplitudes.

In Fig. 2 unstable behaviour of a few particles in the longitudinal phase space is shown for an equipartitioned bunched beam by exciting a pure $20 \%$ transverse, $30 \%$ longitudinal high mode with an analytical phase advance of $166^{\circ}$. The transverse and longitudinal tune depressions are 0.77 . The longitudinal zero current tune is about $90^{\circ}$ and the transverse zero current tune is about $85^{\circ}$. The bunch is almost spherical. No unstable behaviour has been observed in the transverse planes. The tune of the most unstable particle is shown in Fig. 3. Its value is close to $90^{\circ}$ after 50 periods.

Both transverse emittances of the longitudinally most unstable particles are close to the transverse rms emittances. Similar correlations for transversely outermost particles have been observed at the end of the ESS linac [5]. Comparing Fig. 2 with Fig. 1 the difference between an envelope resonance and unstable behaviour due to resonance overlap is evident. Few particles are expelled to very large emittance values when their tune is close to $90^{\circ}$.

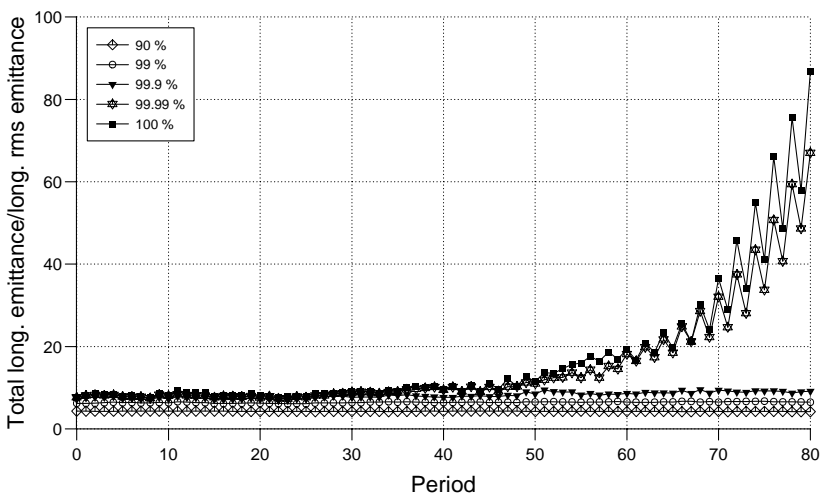

Figure 2: Longitudinal emittance ratio along a mismatched bunched beam transferline

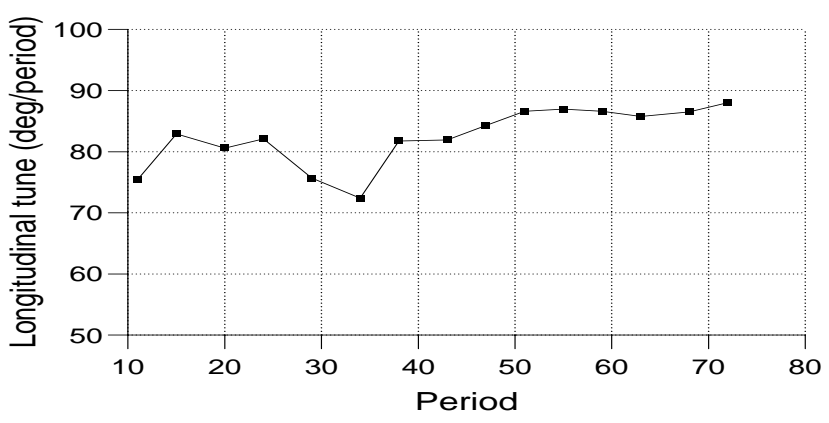

Figure 3: Single particle tune of the most unstable particle which is close to $90^{\circ}$ after 50 periods

\subsection{Unstable Particle Motion due to Tempera- ture Exchange}

In the design of high intensity linear accelerators substantial differences between transverse and longitudinal temperatures should be avoided [6,7].

In Fig. 4 the development of unstable particle motion due to temperature exchange and leading to the $90^{\circ}$ particle - lattice resonance is shown for a matched beam. The longitudinal to transverse temperature ratio is 3 and the zero current transverse tune is $92^{\circ}$. The not shown rms emittances are oscillating due to temperature exchange. Clearly visible is the unstable motion of particles leading to very large emittances in the horizontal phase plane. No unstable behaviour is seen in the longitudinal plane. The single particle tune of $90^{\circ}$, as shown in Fig. 5, for the least stable particle, is in resonance with the periodic focusing, leading to the strong increase in its emittance value. Before the particle becomes unstable in one transverse plane, energy transfer between the longitudinal and the transverse plane is observed.

\subsection{Unstable Particle Motion due to Large Mis- match Amplitudes}

By avoiding temperature exchange, the $90^{\circ}$ single particle - lattice resonance and high tune depression, mismatch amplitudes smaller than $30 \%$ lead to a superposition of 3 


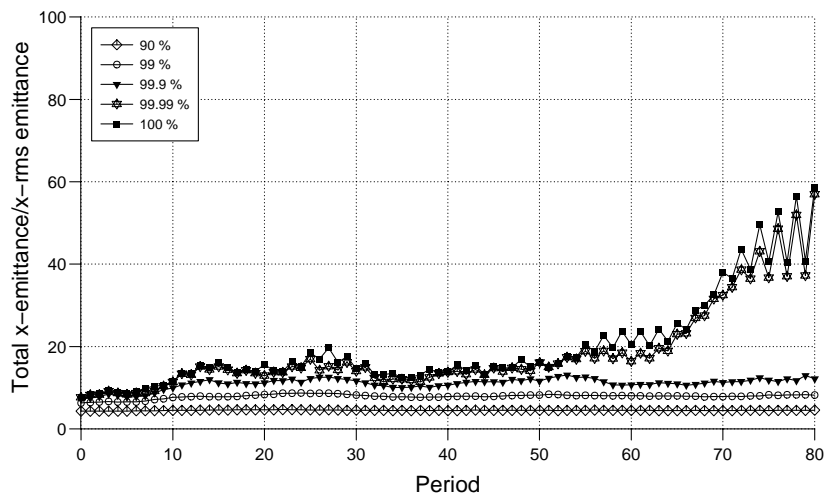

Figure 4: Transverse emittance ratio along a matched transfer line with temperature exchange

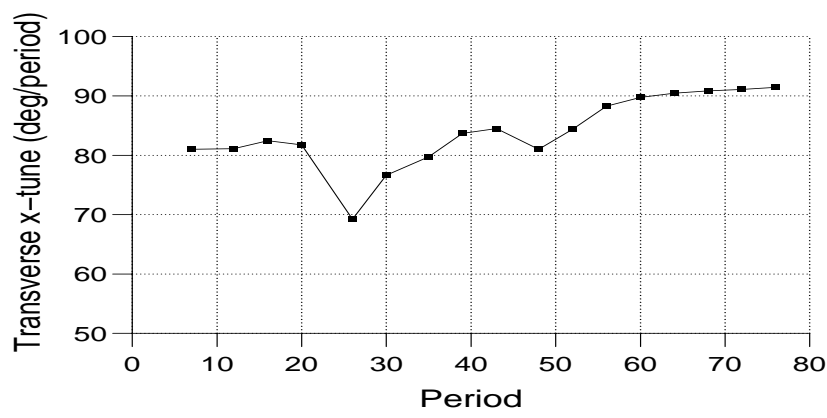

Figure 5: Single particle tune of the most unstable particle which is close to $90^{\circ}$ after 50 periods

bunched beam eigenmodes.

The situation can be different for mode excitations with large amplitudes. For the layout of the periodic focusing system in Fig. 1, a 20\% 'pseudo' high mode (equal mismatch amplitudes in all three directions) excitation leads to neither transverse nor longitudinal halo formation. By $50 \%$ pseudo high mode excitation however, mode overlap occurs and leads to unstable particle motion in all three planes after 40 periods. The periodic focusing system enlarges the maximal halo extent compared to an uniform focusing channel.

\section{MONTE CARLO SIMULATION OF THE ESS LINAC}

All the results above are for a bunched beam transfer line with no acceleration. The conclusions are also valid for the design of a high current linac. As an example a 50000 particle Monte Carlo simulation result is shown for the $214 \mathrm{~mA}$ ESS coupled cavity linac which accelerates the beam from $70 \mathrm{MeV}$ up to $1.334 \mathrm{GeV}$ [8]. The injection parameters at $70 \mathrm{MeV}$ are about the same as for the discussed transferline in Fig. 4. The ratio between full and zero current tunes is greater than 0.7 both transversely and longitudinally, all along the linac. The transverse zero current tune is $92^{\circ}$ at injection.

The ratio between the transverse and longitudinal tem- perature in the rest system is 0.36 at injection and at linac end. The maximum value of 0.5 is reached about $360 \mathrm{MeV}$.

For the matched case there are no particles outside $15 * \epsilon_{r m s}$ either transversely or longitudinally and the rms emittances change by about $20 \%$ due to temperature exchange. Fig. 6 shows the halo formation due to exciting a pure high mode with $20 \%$ transverse and $20 \%$ longitudinal mismatch.

As the $90^{\circ}$ particle - lattice resonance is crossed transversely together with increasing the transverse temperature, halo formation due to mismatch is visible in the horizontal plane. Much weaker halo formation is seen in the longitudinal phase plane as expected from the high mode excitation.

These results are very much consistent with the results for a bunched beam in a periodic focusing channel. The unstable particle motion in the transverse plane however is much less dominant compared to similar periodic transport line of Fig. 4, because the very dangerous parameter combination of temperature ratio of 0.37 together with $\sigma_{t}^{o}=92^{\circ}$ is crossed quite fast due to acceleration. Fast crossing of the $90^{\circ}$ particle - lattice resonance therefore is a possibility for designing high intensity linacs if equipartitioning would lead to strong tune depression.

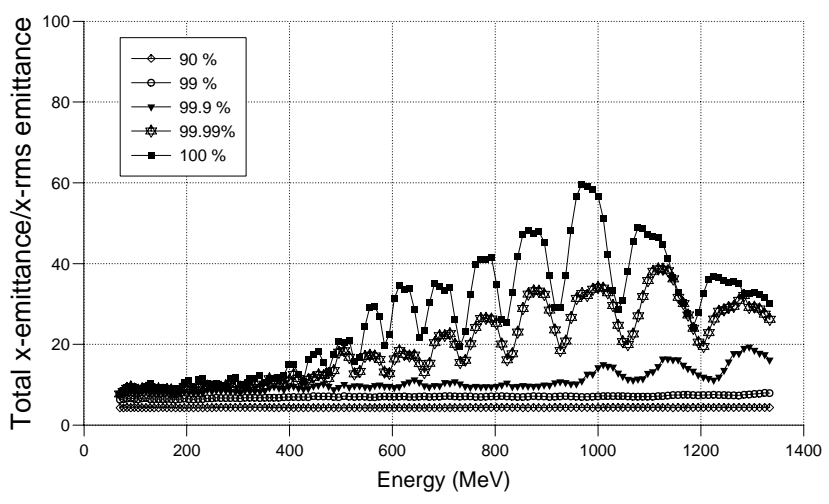

Figure 6: Transverse emittance ratio along the ESS linac for $20 \%$ high mode excitation

\section{REFERENCES}

[1] M. Pabst et al., "Progress on Intense Proton Beam Dynamics", Proc. EPAC 1998, Stockholm, Sweden, p. 146

[2] R. L. Gluckstern et al. Phys. Rev. E 58, 1994, p. 4977

[3] J. Struckmeier et al., Part. Acc., vol. 15, p. 47, 1984

[4] K. Bongardt et al., "Halo Formation by Mismatch for High Intensity Bunched Beams", Proc. LINAC 1998, Chicago, USA

[5] M. Pabst et al., Proc. PAC 1997, Vancouver, Canada, p. 1846

[6] M. Reiser, "Theory and Design of Charged Particle Beams", John Wiley, New York, 1994

[7] R. A. Jameson, Fusion Eng. Design, 32-33, p. 149, 1996

[8] "The European Spallation Source Study", Vol. 1-3, march 1997 